\section{Diagnostic value of ultrasound halo count and Halo Score in giant cell arteritis: a retrospective study from routine care}

We read with great interest the paper published by van der Geest et $a l^{1}$ on 'Novel ultrasonographic Halo Score for giant cell arteritis (GCA): assessment of diagnostic accuracy and association with ocular ischaemia'. The authors aimed to quantify the extent of vascular inflammation by ultrasound (US) in patients with GCA and developed two novel US scoring systems, the halo count and Halo Score, including the assessment of the three temporal artery (TA) segments and axillary arteries. First, we would like to congratulate them for the novelty of their work that opens up new perspectives in the use of US in the assessment of GCA. According to recent EULAR recommendations, US is recommended as the first imaging modality in patients with suspected predominantly cranial GCA. ${ }^{2}$ The halo sign is the most relevant US finding in GCA and is defined as a homogeneous, hypoechoic wall thickening, well delineated towards the luminal side, visible in two perpendicular planes, most commonly concentric in transverse scan. ${ }^{3}$ The halo count and Halo Score constitute the first quantitative tools to assess the extent of vascular inflammation by US in GCA. ${ }^{1}$ According to their findings, a high volume of vascular inflammation on US might strongly support the diagnosis of GCA, is linked to systemic markers of inflammation and identifies patients at risk for ocular ischaemia. On the other hand, a modified Halo Score has been recently proposed by Chattopadhyay et $a l^{4}$ including the assessment of three vascular territories (bilateral temporal, subclavian and axillary arteries) instead of two, as the original Halo Score may underestimate the burden of the inflammation in large-vessel GCA and Takayasu arteritis.

We aim to assess the diagnostic value of both scoring systems and its association with systemic inflammation in patients with GCA seen in routine care. This was a retrospective observational study including patients suspected of having GCA over a 9-month period. All patients underwent bilateral US examination of the three TA segments (common superficial TA, its parietal and frontal branches) and extracranial (carotid, subclavian and axillary) arteries as part of a diagnostic fast track pathway (FTP) $)^{5}$ where US is undertaken within 24 hours. The extent of vascular inflammation was quantified according to the halo count (number of TA segments and axillary arteries with a halo) ranging from 0 to 8 and the Halo Score (a composite index that incorporates both the number of halos and the maximum halo thickness in each region) ranging from 0 to $48 .{ }^{1} \mathrm{TA}$ biopsy was performed according to the treating clinician criteria. The gold standard for GCA was the clinical diagnosis after 6 months of follow-up. Validity was analysed by receiver operating characteristic (ROC) curves and correlations were determined by Spearman's rank correlation coefficient (rho).

Fifty-eight patients were evaluated in the FTP (mean age 74.7 years, $65.5 \%$ females). Clinical and US variables of patients with and without GCA are shown in table 1. A clinical diagnosis of GCA was established in 15 (25.9\%) patients. Only 4.7\% patients without GCA versus $86.7 \%$ with GCA had positive US findings according to the ultrasonographer criteria (sensitivity (Sens) $86.7 \%$, specificity (Spec) 95.3\%, positive likelihood ratio (LR+) 18.4 and negative likelihood ratio (LR-) 0.14). Halo count and Halo Score showed similar diagnostic accuracy for a clinical diagnosis of GCA (area under the ROC

Table 1 Clinical, laboratory and ultrasound findings of patients included in the fast track pathway

\begin{tabular}{|c|c|c|c|c|}
\hline & Total, $n=58$ & Patients with GCA, $n=15$ & Patients without $G C A, n=43$ & $P$ value \\
\hline Age, mean (SD) & $74.7(10.9)$ & $76.5(10.2)$ & $74(11.3)$ & 0.431 \\
\hline Sex, no. of female & $38(65.5 \%)$ & $8(53.3 \%)$ & $30(69.8 \%)$ & 0.249 \\
\hline Baseline use of steroids, no. of patients & $28(49.1 \%)$ & $6(40 \%)$ & $22(52.4 \%)$ & 0.410 \\
\hline TA biopsy positive $n=11$, no. of patients & $3(27.3 \%)$ & $3(37.5 \%)$ & $0(0 \%)$ & 0.491 \\
\hline TA biopsy length $(\mathrm{mm}) \mathrm{n}=11$, mean (SD) & $5.5(3.1)$ & $5.9(3.6)$ & $4.7(1.5)$ & 0.6 \\
\hline${ }^{18} \mathrm{~F}-\mathrm{FDG}$-PET/CT positive $\mathrm{n}=10$, no. of patients & $5(50 \%)$ & $4(66.7 \%)$ & $1(25 \%)$ & 0.197 \\
\hline Fulfilling 1990 GCA criteria, no. of patients & $13(22.4 \%)$ & $5(33.3 \%)$ & $8(18.6 \%)$ & 0.239 \\
\hline Headache, no. of patients & $30(51.7 \%)$ & $11(73.3 \%)$ & $19(44.2 \%)$ & 0.052 \\
\hline Scalp tenderness, no. of patients & $4(6.9 \%)$ & $2(13.3 \%)$ & $2(4.7 \%)$ & 0.273 \\
\hline Jaw claudication, no. of patients & $10(17.2 \%)$ & $7(46.7 \%)$ & $3(7 \%)$ & 0.002 \\
\hline Visual symptoms, no. of patients & $10(17.2 \%)$ & $5(33.3 \%)$ & $5(11.6 \%)$ & 0.055 \\
\hline Fever, no. of patients & $7(12.1 \%)$ & $2(13.3 \%)$ & $5(11.6 \%)$ & 1 \\
\hline Polymyalgia, no. of patients & $27(46.6 \%)$ & $10(66.7 \%)$ & $17(39.5 \%)$ & 0.07 \\
\hline Ocular ischaemia, no. of patients & $3(5.2 \%)$ & $1(6.7 \%)$ & $2(4.7 \%)$ & 1 \\
\hline Abnormal TA clinical examination, no. of patients & $4(6.9 \%)$ & $2(13.3 \%)$ & $2(4.7 \%)$ & 0.273 \\
\hline $\mathrm{CRP}(\mathrm{mg} / \mathrm{dL})$, mean $(\mathrm{SD})$ & $4.5(6.7)$ & $9.3(8.8)$ & $2.7(4.6)$ & 0.001 \\
\hline $\operatorname{ESR}(\mathrm{mm} / \mathrm{h})$, mean $(\mathrm{SD})$ & $51.2(33.7)$ & $65.7(33.2)$ & $46.1(33.1)$ & 0.075 \\
\hline Haemoglobin (g/dL), mean (SD) & $12.6(1.7)$ & $11.9(1.6)$ & $12.9(1.6)$ & 0.05 \\
\hline Platelets $10^{9} / \mathrm{L}$, mean (SD) & $266.8(96)$ & $307.5(104.1)$ & $252.2(89.7)$ & 0.081 \\
\hline Positive US findings, no. of patients & $15(25.9 \%)$ & $13(86.7 \%)$ & $2(4.7 \%)$ & $<0.001$ \\
\hline TA positive US findings, no. of patients & $11(19 \%)$ & $10(66.7 \%)$ & $1(2.3 \%)$ & $<0.001$ \\
\hline Axillary positive US findings, no. of patients & $8(13.8 \%)$ & $7(46.7 \%)$ & $1(2.3 \%)$ & $<0.001$ \\
\hline TA+axillary positive US findings, no. of patients & $4(7 \%)$ & $4(26.7 \%)$ & $0(0 \%)$ & 0.003 \\
\hline Halo sign positive, no. of patients & $15(25.9 \%)$ & $13(86.7 \%)$ & $2(4.7 \%)$ & $<0.001$ \\
\hline Compression sign positive, no. of patients & $8(13.8 \%)$ & $7(46.7 \%)$ & $1(2.3 \%)$ & $<0.001$ \\
\hline Halo count, mean (SD) & $0.7(1.4)$ & $2.5(1.9)$ & $0.04(0.2)$ & $<0.001$ \\
\hline Halo Score, mean (SD) & $4.5(8.7)$ & $15.8(9.9)$ & $0.5(2.7)$ & $<0.001$ \\
\hline
\end{tabular}

CRP, C-reactive protein; ESR, erythrocyte sedimentation rate; 18F-FDG-PET/CT, Fluorine-18 Fluorodeoxyglucose Positron Emission Tomography/Computed Tomography; GCA, giant cell arteritis; TA, temporal artery; US, ultrasound. 
A

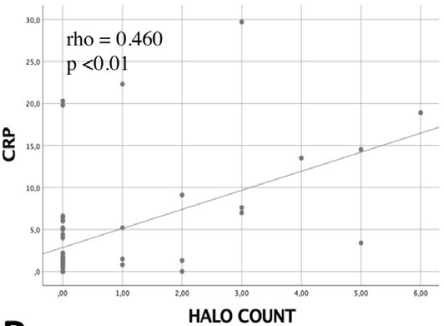

B

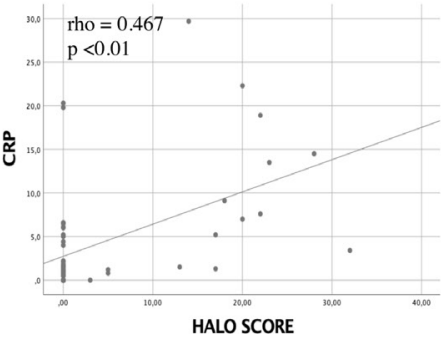

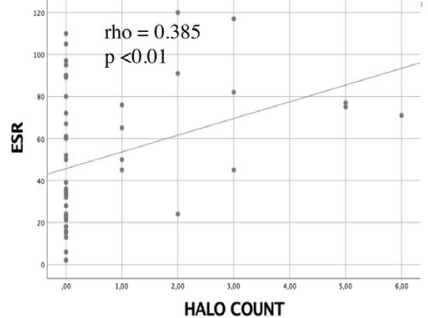

HALO COUNT

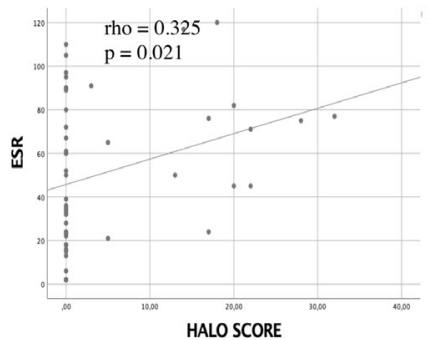

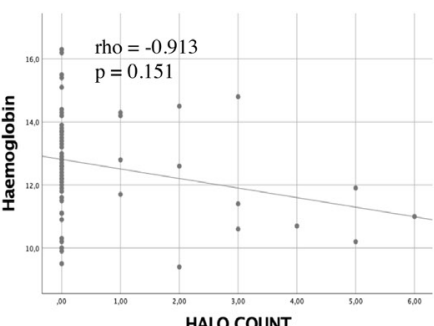

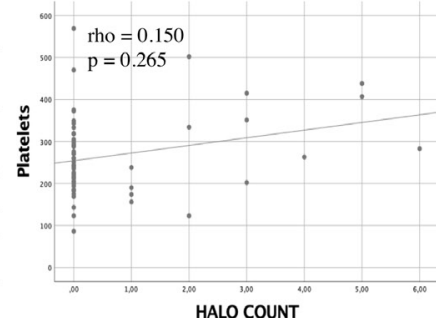

HALO COUNT
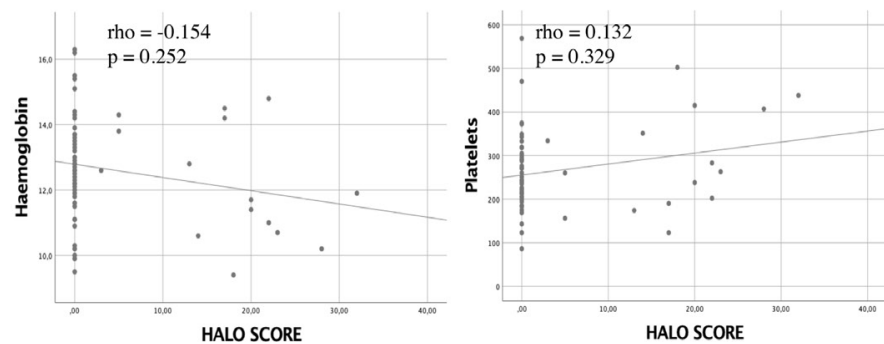

Figure 1 Correlations between halo count (A) and Halo Score (B) with markers of systemic inflammation (CRP, ESR, haemoglobin and platelets). CRP, C-reactive protein; ESR, erythrocyte sedimentation rate.

curve of 0.892 and 0.921 , respectively). The optimal cut-off point for halo count was $\geq 1$ (Sens $80 \%$, Spec 95.3, LR+ 17.02, LR0.21 ) and for Halo Score $\geq 2$ (Sens $86.7 \%$, Spec 95.3\%, LR+18.4, LR - 0.14). Statistically moderate positive correlations were found between halo count and Halo Score and ESR (rho 0.385 and 0.325 , $\mathrm{p}<0.05$ ) and C-reactive protein (CRP) (rho 0.460 and 0.467, $\mathrm{p}<0.01)$, but not with haemoglobin and platelet count $(\mathrm{p}>0.05)$ (figure 1).

To our knowledge, this is the first study to assess the diagnostic value of the halo count and Halo Score in a routine clinical setting, after its first description by van der Geest et al. ${ }^{1}$ They first demonstrated that the Halo Score correlated positively with CRP levels and platelet counts and negatively with haemoglobin levels, but they found no correlation with ESR. Our findings confirm the link between both scoring systems with systemic inflammation in GCA, both with CRP and ESR, and show a good diagnostic accuracy in a clinical setting. In summary, the extent of vascular inflammation by US halo count and Halo Score can help to support the diagnosis of GCA in routine care as they correlate with laboratory markers of systemic inflammation. In the future, they may also have a role in monitoring disease activity. Although both scoring systems needs further validation, they can be easily implemented in FTP of patients with GCA.

Juan Molina Collada ๑ , Julia Martínez-Barrio, Belén Serrano-Benavente, Isabel Castrejón, Liz Rocío Caballero Motta, Laura Trives Folguera, José María Álvaro-Gracia (0)

Rheumatology, Hospital General Universitario Gregorio Marañón, Madrid, Spain

Correspondence to Juan Molina Collada, Rheumatology, Hospital General Universitario Gregorio Marañón, Madrid 28007, Spain; molinacolladajuan@gmail.com

Twitter Juan Molina Collada @jmolinacollada

Acknowledgements The authors thank all the patients who participated in this study.

Contributors All authors made substantial contributions to the conception and design of this study. Study design was performed by JMC and IC. Subject recruitment and US examination were performed by JMC. JMC and LRCM collected the epidemiological and clinical data. JMC and IC performed the statistical analysis. JMC, JM-B, BS-B, IC, LRCM, LTF and JMA-G drafted the manuscript. All coauthors revised the final manuscript.

Funding The authors have not declared a specific grant for this research from any funding agency in the public, commercial or not-for-profit sectors.
Competing interests None declared.

Patient and public involvement Patients and/or the public were not involved in the design, or conduct, or reporting or dissemination plans of this research.

Patient consent for publication Not required.

Ethics approval The research protocol has been approved by the Research ethical Committee of Hospital General Universitario Gregorio Marañón, and all patients gave informed written consent for their participation in the study.

Provenance and peer review Not commissioned; internally peer reviewed.

(c) Author(s) (or their employer(s)) 2020. No commercial re-use. See rights and permissions. Published by BMJ.

\section{Check for updates}

To cite Molina Collada J, Martínez-Barrio J, Serrano-Benavente B, et al. Ann Rheum Dis Epub ahead of print: [please include Day Month Year]. doi:10.1136/ annrheumdis-2020-218631

Received 17 July 2020

Accepted 18 July 2020

\section{SLinked}

- http://dx.doi.org/10.1136/annrheumdis-2020-218654

Ann Rheum Dis 2020;0:1-2. doi:10.1136/annrheumdis-2020-218631

ORCID iDs

Juan Molina Collada http://orcid.org/0000-0001-5191-7802

José María Álvaro-Gracia http://orcid.org/0000-0002-0343-3747

\section{REFERENCES}

1 van der Geest KSM, Borg F, Kayani A, et al. Novel ultrasonographic halo score for giant cell arteritis: assessment of diagnostic accuracy and association with ocular ischaemia. Ann Rheum Dis 2020;79:393-9.

2 Dejaco C, Ramiro S, Duftner C, et al. EULAR recommendations for the use of imaging in large vessel vasculitis in clinical practice. Ann Rheum Dis 2018;77:636-43.

3 Bruyn GA, lagnocco A, Naredo E, et al. OMERACT definitions for ultrasonographic pathologies and elementary lesions of rheumatic disorders 15 years on. J Rheumatol 2019:46:1388-93.

4 Chattopadhyay A, Ghosh A. 'Halo Score': missing large-vessel giant cell arteritis- do we need a 'modified Halo Score'? Ann Rheum Dis 2020;annrheumdis-2020-218224.

5 Diamantopoulos AP, Haugeberg $\mathrm{G}$, Lindland A, et al. The fast-track ultrasound clinic for early diagnosis of giant cell arteritis significantly reduces permanent visual impairment: towards a more effective strategy to improve clinical outcome in giant cell arteritis? Rheumatology 2016:55:66-70. 